\title{
El mercado nacional de fórmulas para la alimentación infanti
}

Pilar Torre ${ }^{1 *}$ y Monserrat Salas ${ }^{2}$

${ }^{1}$ Universidad Autónoma Metropolitana Unidad Xochimilco, Departamento de Atención a la Salud; ${ }^{2}$ Instituto Nacional de Ciencias Médicas y Nutrición "Salvador Zubirán", Departamento de Estudios Experimentales y Rurales. Ciudad de México, México

La lactancia materna es la modalidad alimentaria óptima para el crecimiento, desarrollo y salud infantiles, debe ser exclusiva durante los primeros seis meses de vida y con alimentación complementaria hasta los 24 meses. En México, la prevalencia de lactancia materna exclusiva entre los menores de seis meses es de $31 \%,{ }^{1}$ pero no se cuenta con información del consumo de fórmulas para la alimentación infantil o de otros alimentos.

En 2016, en 35 establecimientos comerciales en la Ciudad de México se encontraron 79 nombres de fórmulas para la alimentación infantil; 57 fueron importadas. Los precios por lata de $400 \mathrm{~g}$ fluctuaron entre 5 y 33.4 dólares. En el mismo año, el volumen de las ventas nacionales fue de 78 mil toneladas, con valor de 596 millones de dólares. Esta cantidad generaría 600 millones de litros de fórmula preparada,
$743 \mathrm{~mL}$ por nacido vivo por día, que permîiría alimentar únicamente con fórmulas a todos los nacidos vivos en ese año en México. ${ }^{2}$

Las empresas ofrecen disponibilidad "universat" de fórmulas para la alimentación infantil y constrựen una episteme que las presenta como equivalentes a la lactancia materna mediante discursos de funciönarios, académicos y una compleja mercadotecnia. La discusión ética sobre el conflicto de intereses en la alimentación infantil resulta imperativa.

\section{Bibliografía}

1. Fondo de las Naciones Unidas para la Infancia/Instituto Nacionál de Salud Pública. Encuesta Nacional de Niños, Niñas y Mujeres 20 t5. Informe final. México: Fondo de las Naciones Unidas para la Infancia/ Instituto Nacional de Salud Pública; 2016.

2. Torre $P$ Salas $\mathrm{M}$, Silva $\mathrm{Cl}$. Desafíos para la promoción de la sa:日ed el caso del mercado de las fórmulas infantiles en México. Glob Health Promot. 2019:1-9.
Gac Med Mex. 2019;155:656

Disponible en Pứbled www.gacetamedicademexico.com 